\title{
Geração Y e Educação Financeira
}

\author{
Derson da Silva Lopes-Jr. ${ }^{1}$ \\ Ivam Ricardo Peleias ${ }^{2}$
}

\begin{abstract}
Resumo
Este trabalho analisou o nível de conhecimento financeiro de jovens da geração $\mathrm{Y}$ e a influência dos fatores faixa etária, gênero e curso no resultado obtido. Para realização do estudo, foi selecionada uma amostra de 464 estudantes de um centro universitário na zona sul de São Paulo. Foi aplicado um questionário adaptado a partir do estudo de Lusardi e Mitchell (2006). Após análise dos dados pela estatística descritiva e aplicação do método de regressão logística binária, concluiu-se que esse grupo de jovens possui baixo nível de conhecimento financeiro, resultado alcançado por $73,06 \%$ dos respondentes. Além disso, a variável gênero foi significante, apontando uma maior probabilidade de conhecimento financeiro alto/moderado entre os homens. Os resultados dessa pesquisa podem contribuir para as instituições financeiras e também de crédito, auxiliando no processo de moldar os produtos e serviços oferecidos à geração Y; para os governos, demonstrando a necessidade de orientar os jovens quanto a questões financeiras; para os jovens dessa geração, alertando-os quanto a necessidade de ampliar suas bases de conhecimento financeiro e para as empresas em geral que oferecem produtos e serviços para os jovens, demonstrando a forma como esse grupo administra suas finanças, fator importante para avaliação do potencial de consumo.
\end{abstract}

Palavras-Chave: Educação Financeira. Geração Y. São Paulo.

\section{Millennial Generation and Financial Literacy}

\begin{abstract}
This paper analyzed the level of financial knowledge of millennials and the influence of age factors, gender and course degrees at the result. To conduct the study we selected a sample of 464 students attending a university in the southern area of São Paulo. We applied a questionnaire adapted from Lusardi and Mitchell (2006). After the descriptive statistics we analyzed data through binary logistic regression method concluding that the majority of 73,6 percent of this group had a low level of financial knowledge. In addition, the gender variable was significant, indicating a greater probability of high/moderate financial knowledge level among men. The study may contribute to financial and credit institutions, assisting them in the process of modelling products and services rendered to the millennials; for governments, demonstrating the need to address young people about financial matters; for the younger generation, warning them about the need to expand their financial knowledge; and for businesses in general that offer products and services for young people, demonstrating how this group manages its finances, an important factor to assess the potential consumption.
\end{abstract}

Keywords: Financial Education. Millennials. Sao Paulo

\section{INTRODUÇÃO}

\footnotetext{
1 Doutorando em Administração na FEA/USP - Faculdade de Administração, Economia e Contabilidade Universidade de São Paulo. dersonlopes@usp.br

2 Doutor em Controladoria pela FEA/USP. ivar@fecap.br
} 
A importância da educação financeira integra o senso comum. Ela é útil para uso ou compra de produtos financeiros mais sofisticados e para cálculos financeiros do cotidiano, tais como elaborar um orçamento familiar considerando a capacidade de pagamento de juros, o pagamento do cartão de crédito / débito ou avaliar os preços dos serviços públicos (SAVOIA; SAITO; SANTANA, 2007).

A educação financeira evoluiu rapidamente na última década no Brasil. No início, as ações eram isoladas e dependiam de algumas entidades que as promoviam. Posteriormente, foi criada a ENEF (Estratégia Nacional de Educação Financeira) que propiciou uma rápida expansão dos conceitos e de iniciativas de disseminação do conhecimento financeiro para vários segmentos da população (BRASIL, 2010). Esta evolução foi corroborada por dois fenômenos sociais: a inclusão financeira de mais de 30 milhões de brasileiros nos últimos anos que anteriormente não estavam inseridos no universo bancário e a afluência da classe média, estimada atualmente em mais de 70 milhões de brasileiros.

Além das mudanças citadas, as novas gerações têm demonstrado valores e perfis distintos dos encontrados no passado, especialmente a geração Y, que atualmente se fortalece na sociedade e no mercado de trabalho. Para essa geração, a busca por qualidade de vida, liberdade e flexibilidade está gradualmente substituindo os valores fundamentais do trabalho e da segurança de um emprego vitalício (SCHIFFMAN; KANUK, 2007; CENNAMO; GARDNER, 2008; FILENGA; VIEIRA, 2012). Motivações de consumo, percepção de risco e de valor do dinheiro estão mudando gradualmente, e, em alguns casos essa mudança traz efeitos negativos para a sociedade (TWENGE; CAMPBELL, 2008; HERBIG; BORSTORFF, 1995; HEANEY, 2007).

Apesar de diversos esforços empreendidos por entidades públicas e privadas, o nível de endividamento entre os jovens é crescente (MANDELL, 2008). Cerca de um quarto das falências pessoais declaradas no mundo são de jovens com até 25 anos de idade (SHRYK, 2008). Na busca de uma solução para esse problema, Lyon (2004) afirma que jovens sem educação financeira se tornam mais vulneráveis ao desequilíbrio em suas contas e Lyon et al. (2006) destacam que o conhecimento financeiro pode atuar como medida preventiva contra o endividamento dos jovens, sendo, portanto, importante estudar maneiras de educa-los nesta área.

Diante da relevância do tema apresentado, o presente artigo pretende contribuir para o debate quanto à avaliação da educação financeira e sua incidência entre os jovens. $O$ trabalho está estruturado da seguinte fora: além dessa introdução, a revisão bibliográfica é apresentada 
na seção um, os princípios metodológicos na seção dois, os resultados e suas respectivas análises na terceira seção e por fim as considerações finais e referências bibliográficas.

\section{REVISÃO BIBLIOGRÁFICA}

A educação financeira é uma preocupação crescente em vários países, o que tem gerado um aprofundamento nos estudos sobre esse tema. Existem críticas quanto à abrangência dos programas existentes e seus resultados, bem como análises que indicam ser a experiência relacionada a vida financeira mais impactante do que a educação financeira (LYON; CHANG; SCHERPF, 2006), no entanto é inegável a importância do desenvolvimento de ações planejadas de habilitação da população nesta área de estudos (OCDE, 2005).

Agarwal et al. (2009) concluíram que muitos adultos não são educados financeiramente e esse déficit no conhecimento financeiro leva a tomada de decisões não otimizadas. Lusardi e Mitchell (2007) afirmam que a falta de conhecimento financeiro é um mal presente em diversos países. Os indivíduos possuem dificuldades para a elaboração de seu planejamento financeiro, o que compromete o processo de acumulação de riqueza para a aposentadoria.

Estudos têm revelado que o aumento do conhecimento financeiro tem um impacto positivo nas tomadas de decisões financeiras mais vantajosas para as pessoas (MANDELL; KLEIN, 2009). Apesar disso, nem todos estão interessados em adquirir esse conhecimento. Os governos e o setor privado enfrentam desafios para motivar as pessoas a adquirir educação financeira (HILGERT; HOGARTH; BEVERLY, 2003).

A Organização para Cooperação do Desenvolvimento Econômico (OCDE) criou em 2003 um projeto para estudar a educação financeira e propor programas aos seus paísesmembros. Observou-se que os países pesquisados estão adotando políticas para instruir a população nos conceitos de crédito, de investimentos e de instrumentos de seguro (OCDE, 2005). As iniciativas empreendidas têm sido de conhecimentos básicos de finanças, uma vez que é custoso e de sucesso improvável transformar pessoas sem qualquer conhecimento financeiro em investidores profissionais; porém, pode-se ensinar-lhes as noções básicas de controle financeiro, poupança e investimento (VAN ROOIJ; LUSARDI;ALESSI, 2011).

\subsection{Definições}

Há várias definições para a expressão educação financeira. Para a OCDE, ela é uma combinação de consciência, conhecimento, habilidade, atitude e comportamento necessários 
para que as pessoas tomem decisões financeiras e com isso alcancem seu bem-estar financeiro individual (OCDE, 2011).

Remund (2010) afirma que educação financeira é a medida do grau em que um indivíduo entende conceitos financeiros chave e possui a habilidade e confiança para administrar de forma apropriada suas finanças pessoais, por meio de decisões de curto-prazo e planejamento financeiro de longo prazo, em meio aos eventos que ocorrem em sua vida e às mudanças de condições econômicas.

A educação financeira pode ser definida também como a aplicação dos conhecimentos básicos sobre juros compostos, no contexto cotidiano das escolhas financeiras. Possuir educação financeira significa que os indivíduos apresentam habilidades ao fazer escolhas adequadas para administrar suas finanças pessoais durante o ciclo de sua vida (HUSTON, 2010).

\subsection{Determinantes e mensuração do conhecimento financeiro}

Apesar dos esforços aplicados para padronizar a mensuração do conhecimento financeiro, não há consenso no meio acadêmico sobre os melhores instrumentos para mensurar a educação financeira dos cidadãos (PORTRICH; VIEIRA; CERETTA, 2013). Esta é uma questão complexa por sua subjetividade, o que resulta em diversidade de interpretações. Lusardi e Mitchell (2011a) defendem que, embora seja importante avaliar o nível de educação financeira das pessoas, na prática, é difícil explorar a forma como elas processam as informações financeiras e tomam decisões baseadas nessas informações. Para Huston (2010), seria importante determinar se a pessoa tem a informação, bem como se sabe aplicá-la de forma apropriada.

A abordagem econômica convencional de poupança e decisões de consumo afirma que um indivíduo racional e bem informado vai consumir menos de sua renda em tempos de altos rendimentos e economizar para apoiar o consumo quando houver queda de sua renda, por exemplo, após a aposentadoria (LUSARDI, 2009). Uma pessoa educada financeiramente deve determinar seus padrões ideais de poupança, visando o uso dos recursos no decorrer dos anos em função da diminuição de sua atividade produtiva e rentável. (LUSARDI; MITCHELL, 2013).

Nos últimos anos, foram realizados diversos trabalhos sobre o tema e suas implicações em outras questões comportamentais e de consumo, dos quais se destacam: Chen e Volpe (2002), Norvilitis, Szablicki e Wilson (2003), Avard, Manton, English e Walker (2005), Lusardi e Mitchell (2006), Norvilitis et al. (2006), Murphy e Yetmar (2010), Neidermeyer e 
Neidermeyer (2010), Lusardi e Mitchell (2011b) e Lusardi e Mitchell (2013) nos EUA, Lusardi e Tufano (2009) e Disney e Gathergood (2011) no Reino Unido e Van Rooij, Lusardi e Alessie (2011) na Holanda. No Brasil, o tema também tem sido pauta de estudos: Lucci, Zerrenner, Verrone e Santos (2006), Roger, Favato e Securato (2008), Claudino, Nunes e Silva (2009), Vieira, Bataglia, Sereia, Ribeiro e Lohmann (2009), Portrich, Vieira e Ceretta (2013), Diniz, Pereira e Rogers (2014), entre outros.

Os estudos de Lusardi e Mitchell (2006) buscaram determinar questões que procuram medir o nível de educação financeira em pesquisas norte-americanas. O primeiro teste das pesquisadoras foi desenhar um módulo especial sobre educação financeira para o Health and Rent Study (HRS) (estudo sobre saúde e aposentadoria), de 2004.

Lusardi e Mitchell (2006) desenvolveram três perguntas para o HRS que medem conceitos básicos e fundamentais sobre educação financeira, tais como o funcionamento das taxas de juros, os efeitos da inflação, e a diversificação de risco. As questões desenvolvidas para a avaliação da educação financeira são apresentadas no quadro 1:

Quadro 1 - Questões Originalmente Usadas por Lusardi e Mitchell

\begin{tabular}{|c|l|}
\hline Questão & \multicolumn{1}{|c|}{ Enunciado } \\
\hline 1 & $\begin{array}{l}\text { Suponha que você tinha US\$ 100 em uma conta de poupança e a taxa de juros é } \\
\text { de } 2 \% \text { ao ano. Após 5 anos, quanto você acha que teria na conta se deixou o } \\
\text { dinheiro para crescer: mais de US \$ 102, exatamente US \$ 102, a menos de } 102\end{array}$ \\
\hline 2 & $\begin{array}{l}\text { Imagine que a taxa de juros em sua conta de poupança foi de 1\% ao ano e a } \\
\text { inflação foi de 2\% ao ano. Depois de 1 ano, você seria capaz de comprar mais do } \\
\text { que, exatamente a mesma, ou menos do que hoje, com o dinheiro desta conta? }\end{array}$ \\
\hline 3 & $\begin{array}{l}\text { Você acha que a seguinte afirmação é verdadeira ou falsa? "Comprando ações de } \\
\text { uma única empresa geralmente se obtém um retorno mais seguro do que se } \\
\text { investir em um fundo mútuo de ações." }\end{array}$ \\
\hline
\end{tabular}

Fonte: Lusardi e Mitchell (2006).

O estudo demonstrou que a educação financeira tem maior variação em função do grau de escolaridade, gênero e idade. Metade dos entrevistados com educação inferior ao ensino médio respondeu corretamente à pergunta que exige um cálculo simples das taxas de juro, e cerca de $20 \%$ não souberam responder essa pergunta. A maioria das pessoas sem um diploma universitário não sabia ou respondeu incorretamente a pergunta sobre a diversificação de risco. O nível de educação financeira foi particularmente baixo entre as 
mulheres mais velhas, $61,9 \%$ acertaram a questão de cálculo da taxa de juros, em comparação com $74,8 \%$ dos homens e 70,6\% acertaram a questão da inflação, contra $82,2 \%$ dos homens (LUSARDI; MITCHELL, 2006).

Apenas 47,8\% das mulheres sabiam que investir em ações de uma única empresa é mais arriscado do que um fundo mútuo de ações, contra 59,2\% dos homens. As mulheres foram mais propensas a responder que não sabiam a resposta às três perguntas, com uma fração maior para a questão sobre diversificação de risco, $40 \%$ versus $25 \%$ para os homens (LUSARDI; MITCHELL, 2006). Baixos níveis de educação financeira entre as mulheres são confirmados em outras pesquisas que abrangem grupos mais jovens ou representativos da população (VAN ROOIJ; LUSARDI; ALESSIE, 2011). Um estudo americano dedicado a analisar jovens, denominado National Longitudinal Survey of Youth, incluiu em sua edição de 2007 e 2008 as mesmas questões do estudo da HSR. Foram estudados 7.138 formulários respondidos por jovens de 23 a 28 anos. Os resultados encontrados revelaram um baixo nível de conhecimento financeiro entre os jovens (LUSARDI; MITCHELL; CURTO 2010).

As questões de Lusardi e Mitchell (2006) foram adicionadas à outras pesquisas de diversas naturezas, tanto nos EUA como em outros países: Educação financeira das mulheres (LUSARDI; MITCHELL, 2008); Painel de RAND American Life (ALP), abrangendo todas as idades (LUSARDI; TUFANO, 2009); Capacidade financeira em 2009 com grupos diversificados (LUSARDI; MITCHELL, 2011a); OECD/INFE international pilot exercise, estudo com 12 países membros da OCDE (OCDE, 2011); Estudo sobre educação financeira de etnias da Nova Zelândia (CROSSAN; FESLIER; HURNARD, 2011); Influencia do nível de educação financeira nas decisões sobre o mercado de capitais (VAN ROOIJ; LUSARDI; ALESSIE, 2011); Educação financeira e crise financeira (KLAPPER; LUSARDI; PANOS, 2011), entre outros. Em todas as pesquisas citadas, os resultados encontrados indicaram que em geral os entrevistados possuem um nível de educação financeira baixo ou mediano.

No Brasil, a pesquisa de Portrich, Vieira e Ceretta (2013) avaliou o nível de educação financeira de acadêmicos de uma universidade gaúcha. Foi construído um fator a partir da média da pontuação obtida pelos participantes do estudo em dois conjuntos de questões adaptadas de Van Rooij, Lusardi e Alessie (2011).

Os resultados demonstraram que os participantes possuem um nível baixo de conhecimento financeiro. $\mathrm{Na}$ média, $43,63 \%$ das questões básicas foram respondidas corretamente e 51,69\% das avançadas, inferior aos $60 \%$ definidos como linha de corte para determinação do nível de educação financeira. Cabe ressaltar que apenas 1,3\% dos estudantes acertaram todas as questões (PORTRICH; VIEIRA; CERETTA (2013). A tendência obtida 
por Lusardi e Mitchell (2011b) na pesquisa da HSR foi mantida nessa pesquisa.

Outras características comportamentais, tais como a impulsividade, preferências incomuns ou circunstâncias externas podem contribuir para uma aparente decisão financeira equivocada (CHINEN; ENDO, 2012). Assim, os estudiosos não devem assumir que as pessoas com situações financeiras ruins não possuem necessariamente uma boa educação financeira.

\subsection{Geração Y e as finanças}

O conceito de geração Y (ou millennials) tem sido utilizado para determinar os jovens dos anos 2000. Diferentes padrões sido usados para determinar exatamente quem são as pessoas enquadradas nessa geração baseados em seu ano de nascimento (CORDEIRO et al., 2012). Para conceituar e classificar as gerações no contexto brasileiro é necessário analisar os fatos históricos e sociológicos que impactaram o país. Através dessa análise pode-se fazer uma interpretação dos períodos e as correspondências entre as gerações na sociedade americana e na sociedade brasileira (CORDEIRO et al., 2012). Analisando cuidadosamente os critérios apresentados, Cordeiro et al. (2012) classificam como geração Y no Brasil todos os nascidos entre 1981 e 1999.

Esse grupo busca desafios intelectuais, precisa ter sucesso, se esforça para fazer a diferença, e procura por empregadores que aumentem seu desenvolvimento pessoal e profissional. (NAYYAR, 2001; WILLIAMS; PAGE, 2011). Allen (2004) acrescenta que estabelecer e alcançar objetivos pessoais é mais importante para eles que os ganhos financeiros, demonstrando que em sua perspectiva, fazer uma grande quantidade de dinheiro parece ser menos fundamental do que contribuir para a sociedade e desfrutar de uma vida plena e equilibrada.

A educação financeira dos jovens é fundamental à medida que, na idade adulta se deparam com uma variedade de produtos e serviços financeiros, ao mesmo tempo em que embarcam em grandes eventos do ciclo financeiro e de vida, tais como: emprego, morar sozinhos, comprar o primeiro carro, casar-se e começar uma família. A definição de um estilo de vida é influenciada pelos meios de comunicação, que estimulam a disposição dos jovens para o endividamento (CULL; WHITTON, 2011). A extensão do problema da dívida entre os jovens é ilustrada pelo fato de que, nos Estados Unidos entre 1990 e 2000, a dívida média do cartão de crédito dos estudantes saltou $305 \%$ e entre 2004 e 2009 o aumento foi de $74 \%$. Entre 1997 e 2007, a dívida média de empréstimos dos universitários aumentou 58\%, sendo 6\% só entre 2006 e 2007, descontada a inflação (REED, 2008). 
Os resultados dessas pesquisas sugerem que esse aumento considerável no endividamento aumenta a ansiedade entre os jovens e influencia as principais decisões trabalhistas: Lusardi, Mitchell e Curto (2010) entrevistaram jovens entre 23 e 29 anos e constataram que $30 \%$ dos endividados se revelaram frequentemente preocupados; 29\% pararam de estudar ou diminuíram seus gastos com educação e 22\% aceitaram um emprego que não aceitariam se não estivessem endividados. Uma análise dos itens apresentados revela uma necessidade latente e imediata de educação financeira para a geração Y. Comportamentos financeiros positivos extrapolam o âmbito econômico e se traduzem em maior satisfação na vida e na conquista de um melhor desempenho acadêmico (NGA; YOUNG; SELLAPAN, 2010).

\section{METODOLOGIA}

A presente pesquisa utiliza-se de referencial teórico e análise de dados a partir de pesquisa de campo para obtenção de seus objetivos. Quanto ao seu enfoque, a pesquisa é classificada como quantitativa, caracterizada pelo levantamento e mapeamento de dados de uma população específica e oferecendo tratamento estatístico aos dados coletados (GIL, 2002).

A amostra foi definida de maneira aleatória. Foram entrevistados 464 jovens alunos de um centro universitário, selecionados em quatro cursos: Administração, Ciências Contábeis, Psicologia e Educação Física. Em decorrência do objetivo da pesquisa, foram selecionados os alunos classificados na geração Y dentro do contexto brasileiro, nascidos entre 1981 e 1999.

A instituição de ensino superior escolhida (IES) foi o Centro Universitário Adventista de São Paulo - UNASP Campus SP, localizado no bairro Jardim IAE, próximo ao Capão Redondo, na Zona Sul de São Paulo. A instituição, que existe há 100 anos, é de caráter confessional, mantida por uma denominação religiosa. A escolha por essa entidade foi intencional e ocorreu devido à acessibilidade e disponibilidade de aplicação dos formulários, apoiada pelos dirigentes para a realização do estudo em suas dependências.

Segundo a secretaria da IES, à época da pesquisa havia cerca de 6.000 alunos distribuídos desde a educação infantil até o mestrado. Cerca de 500 estudantes vivem na IES em regime de internato, 5.400 alunos moram em um raio de até $10 \mathrm{~km}$ e o restante em outras regiões. O nível de renda médio dos estudantes e suas famílias situa-se entre R \$ 800,00 e R \$ 2.000,00. Considerada a tendência dos estudos citados no referencial teórico, esta pesquisa usou como base para o desenvolvimento de seu questionário o estudo de Lusardi e Mitchell (2006), porém, como em outras pesquisas realizadas usando as perguntas de Lusardi e

ReFAE - Revista da Faculdade de Administração e Economia, v. 8, n. 2, p. 17-36, 2017 
Mitchell (2006), foram feitas adaptações para contextualização ao público alvo e ao objetivo da pesquisa.

As perguntas 1 e 2 do artigo de Lusardi e Mitchell (2006) foram usadas com o mesmo princípio, com alteração da moeda de dólar para real (pergunta 1), contextualizadas para a realidade de juros brasileira (pergunta 2) e com o estabelecimento de alternativas para a resposta. No questionário de Lusardi e Mitchell (2006), a pergunta 3, que trata de diversificação de ativos para diminuição de risco, é aplicável aos Estados Unidos, onde o mercado de capitais é maduro e uma realidade na vida dos cidadãos comuns. No Brasil, a realidade é diferente nesse sentido, sendo que não se pode considerar esse item como parte do conhecimento básico de finanças, mas no mínimo intermediário. Assim, a terceira pergunta foi alterada para uma adaptação da pergunta usada por Potrich, Viera e Ceretta (2013) para avaliar conhecimento financeiro básico, que leva em consideração o valor do dinheiro no tempo, conforme texto abaixo. As três questões adaptadas estão descritas no quadro 2:

Quadro 2 - Questões utilizadas na Pesquisa

\begin{tabular}{|c|c|c|}
\hline Questão & Enunciado & Alternativas \\
\hline 1 & $\begin{array}{l}\text { Suponha que você tinha } \mathrm{R} \$ 100,00 \\
\text { em uma conta de poupança e a taxa } \\
\text { de juros de retorno é de } 6 \% \text { ao ano. } \\
\text { Após } 5 \text { anos, quanto você acha que } \\
\text { teria na conta se você deixou o } \\
\text { dinheiro para render: }\end{array}$ & $\begin{array}{l}\text { a) mais de } R \$ 106,00 \\
\text { b) exatamente } R \$ 106,00 \\
\text { c) menos de } R \$ 106,00 \\
\text { d) não sei a resposta }\end{array}$ \\
\hline 2 & $\begin{array}{l}\text { Imagine que a taxa de juros de } \\
\text { retorno em sua conta de poupança } \\
\text { foi de } 6 \% \text { ao ano e a inflação foi de } \\
12 \% \text { ao ano. Depois de } 1 \text { ano, você } \\
\text { seria capaz de comprar com esse } \\
\text { dinheiro: }\end{array}$ & $\begin{array}{l}\text { a) mais itens do que antes } \\
\text { b) exatamente a mesma quantidade de } \\
\text { itens que antes } \\
\text { c) menos itens do que antes } \\
\text { d) não sei a resposta }\end{array}$ \\
\hline 3 & $\begin{array}{l}\text { Suponha que José herde } \mathrm{R} \$ \\
10.000,00 \text { hoje e Pedro herde } \mathrm{R} \$ \\
10.000,00 \text { daqui a } 3 \text { anos. Devido } \\
\text { ao recebimento da herança, quem } \\
\text { terá um valor maior? }\end{array}$ & $\begin{array}{l}\text { a) José } \\
\text { b) Pedro } \\
\text { c) terão a mesma quantia } \\
\text { d) não sei a resposta }\end{array}$ \\
\hline
\end{tabular}


Fonte: Elaborado pelos autores

O nível de educação financeira foi determinado conforme o número de questões respondidas corretamente. Foram considerados com nível baixo de conhecimento financeiro os respondentes que acertaram uma ou duas questões. Os que acertaram as três questões foram considerados com conhecimento financeiro alto/moderado. Aqueles que erraram todas as questões ou responderam que não sabiam as três questões, foram considerados sem nenhum nível de conhecimento financeiro. Além da determinação do nível de conhecimento financeiro dos jovens entrevistados, foi verificada a influência de fatores demográficos e socioeconômicos no nível de conhecimento financeiro encontrado. Essa verificação foi realizada através de análise multivariada pelo modelo de regressão logística binária.

A regressão logística binária é utilizada para descrever o comportamento de uma variável dependente binária e diversas variáveis independentes (Fávero et al., 2009). Aplicase quando existem dois resultados possíveis em relação a variável estudada. Esse método tem preferencia de utilização nesses casos em função de ser mais robusto e similar a regressão simples, possuindo testes estatísticos diretos, habilidade de incorporar efeitos não lineares e oferecer uma vasta gama de diagnósticos (Hair et al., 2010). Este método estatístico utiliza-se de uma variável dependente chamada $\mathrm{Y}$, de valor 0 ou 1, e diversas variáveis independentes chamadas X (Hair et al., 2010). Neste estudo, a variável dependente Y estabelecida foi uma dummy relacionada ao fato do respondente possuir nível de conhecimento financeiro alto/moderado (1) ou não (0), sendo considerados com conhecimento alto/moderado aqueles que acertaram as três questões propostas.

As variáveis independentes foram determinadas de acordo com o objetivo da pesquisa. Potrich, Viera e Ceretta (2013) apresentam como principais variáveis demográficas e socioeconômicas que influenciam o nível de conhecimento financeiro: Gênero, idade, estado civil, escolaridade, curso, renda e etnia. Devido a uma restrição da IES, o fator etnia não foi inserido na pesquisa. Os fatores estado civil, renda e escolaridade foram detectados como altamente homogêneos a partir de dados oferecidos pela secretaria da IES. Desta forma foram selecionadas como variáveis independentes os fatores gênero, idade e curso.

Com as variáveis estabelecidas e baseado no referencial teórico, buscou-se analisar os fatores determinantes do nível de conhecimento dos jovens entrevistados, assumindo-se três hipóteses:

Hipótese 1: A faixa etária influencia o nível de conhecimento financeiro, sendo:

H0 - O nível de educação financeira é influenciado pela faixa etária.

H1 - O nível de educação financeira não é influenciado pela faixa etária.

ReFAE - Revista da Faculdade de Administração e Economia, v. 8, n. 2, p. 17-36, 2017 
Hipótese 2: O gênero influencia o nível de conhecimento financeiro, sendo:

H0 - O nível de educação financeira é influenciado pelo gênero.

H1 - O nível de educação financeira não é influenciado pelo gênero.

Hipótese 3: O curso influencia o nível de conhecimento financeiro, sendo:

H0 - O nível de educação financeira é influenciado pelo curso.

H1 - O nível de educação financeira não é influenciado pelo curso.

Os dados foram processados no software estatístico SPSS e interpretados conforme os resultados encontrados na estatística descritiva e na regressão logística. As análises e resultados obtidos são apresentados e discutidos no tópico seguinte.

\section{ANÁLISE DOS RESULTADOS}

Os entrevistados foram classificados por faixa etária, gênero e curso de graduação que estão matriculados. A tabela 2 demonstra a distribuição da amostra conforme os grupos de classificação. Pode-se notar uma concentração na na faixa etária de 16 a 27 anos (88,15\%), no sexo feminino $(61,85 \%)$ e no curso de administração (40,52\%).

Tabela 2 - Distribuição da Amostra por Grupos de Classificação

\begin{tabular}{l|r|l}
\hline \multicolumn{1}{c|}{ Classificação } & Quantidade & $\%$ \\
\hline $16-21$ anos & 215 & $46,34 \%$ \\
$22-27$ anos & 194 & $41,81 \%$ \\
$28-33$ anos & 55 & $11,85 \%$ \\
Feminino & 287 & $61,85 \%$ \\
Masculino & 177 & $38,15 \%$ \\
Administração & 188 & $40,52 \%$ \\
C. Contábeis & 87 & $18,75 \%$ \\
Ed. Física & 83 & $17,89 \%$ \\
Psicologia & 106 & $22,84 \%$ \\
\hline
\end{tabular}

Fonte: Elaborado pelos autores

\subsection{Análise das respostas por grupo de classificação}

As respostas foram classificadas e organizadas conforme os grupos estabelecidos no estudo. Foram considerados os percentuais de acerto de cada grupo nas questões 1,2 e 3 e o percentual de respondentes com 3, 2, 1 e 0 acertos. Os resultados encontrados são apresentados na tabela 3 . 
Tabela 3 - Análise de Resultados por Grupo de Classificação

\begin{tabular}{|c|c|c|c|c|c|c|c|}
\hline Grupos & Acertos Q1 & Acertos Q2 & Acertos Q3 & $\begin{array}{c}\text { Três } \\
\text { acertos }\end{array}$ & $\begin{array}{c}\text { Dois } \\
\text { acertos }\end{array}$ & $\begin{array}{c}\text { Um } \\
\text { acerto }\end{array}$ & $\begin{array}{l}\text { Nenhum } \\
\text { acerto }\end{array}$ \\
\hline Feminino & $79,44 \%$ & $58,89 \%$ & $25,09 \%$ & $11,15 \%$ & $51,22 \%$ & $25,44 \%$ & $12,20 \%$ \\
\hline Masculino & $84,75 \%$ & $75,71 \%$ & $39,55 \%$ & $27,68 \%$ & $49,15 \%$ & $14,12 \%$ & $9,04 \%$ \\
\hline $16-21$ & $83,26 \%$ & $60,93 \%$ & $30,23 \%$ & $17,67 \%$ & $48,37 \%$ & $25,58 \%$ & $8,37 \%$ \\
\hline $22-27$ & $80,41 \%$ & $64,95 \%$ & $28,35 \%$ & $16,49 \%$ & $51,55 \%$ & $20,62 \%$ & $11,34 \%$ \\
\hline $28-33$ & $81,82 \%$ & $78,18 \%$ & $41,82 \%$ & $23,64 \%$ & $60,00 \%$ & $9,09 \%$ & $7,27 \%$ \\
\hline Administração & $81,38 \%$ & $70,21 \%$ & $32,98 \%$ & $23,94 \%$ & $48,40 \%$ & $15,96 \%$ & $11,70 \%$ \\
\hline C. Contábeis & $93,10 \%$ & $73,56 \%$ & $21,84 \%$ & $17,24 \%$ & $62,07 \%$ & $14,94 \%$ & $5,75 \%$ \\
\hline Ed. Física & $75,90 \%$ & $71,08 \%$ & $32,53 \%$ & $15,66 \%$ & $59,04 \%$ & $20,48 \%$ & $4,82 \%$ \\
\hline Psicologia & $77,36 \%$ & $46,23 \%$ & $25,47 \%$ & $14,15 \%$ & $37,74 \%$ & $37,74 \%$ & $10,38 \%$ \\
\hline Total & $81,47 \%$ & $64,87 \%$ & $29,74 \%$ & $17,24 \%$ & $51,08 \%$ & $21,98 \%$ & $9,70 \%$ \\
\hline
\end{tabular}

Fonte: Elaborado pelos autores

Tang (1992) e Roberts e Jones (2001) revelam que o gênero tem influência no perfil financeiro dos indivíduos. Assim como em outros estudos (LUSARDI; MITCHELL, 2011b; VAN ROOIJ; LUSARDI; ALESSIE, 2011), as mulheres obtiveram um desempenho inferior ao dos homens, demonstrando possuir um menor conhecimento financeiro.

Analisando a tabela 3, percebe-se que a distribuição da quantidade de questões respondidas corretamente apresenta que $27,68 \%$ dos homens acertaram todas as questões, enquanto que apenas $11,15 \%$ das mulheres alcançaram esse desempenho. Além disso os respondentes do sexo masculino obtiveram um percentual maior de participantes com respostas corretas nas três questões individualmente em relação aos resultados obtidos pelos respondentes do sexto feminino.

No grupo relacionado à faixa etária, percebe-se um destaque para aqueles que possuem entre 28 e 33 anos, alcançando um percentual superior de acertos nas questões 2 e 3 (78,18\% e 41,82\% respectivamente) e um número maior de respondentes com um total de 3 acertos $(23,64 \%)$. Os achados do estudo de Portrich, Vieira e Ceretta (2013) indicam que o curso no qual o universitário está matriculado influencia seu nível de educação financeira. Quando analisado o número de acertos de cada questionário de acordo com os cursos dos participantes, observa-se um desempenho superior nos respondentes matriculados no curso de administração, com $23,94 \%$ de respondentes que acertaram todas as questões. Esse dado é 
justificado pelo fato de que os cursos de administração normalmente os alunos tem de 5 até 10 disciplinas de finanças, o que os tornaria mais preparados para administrar suas finanças de forma geral.

Como apresentado na seção referente a metodologia, os estudantes que responderam as três questões corretamente foram considerados com conhecimento financeiro alto/moderado. Aqueles que responderam até duas questões corretamente foram considerados com nível baixo de conhecimento financeiro. Os que responderam de maneira incorreta todas as questões ou responderam que não sabiam as três questões, foram considerados sem nenhum nível de conhecimento financeiro.

Com base nos resultados apresentados na tabela 3, pode-se afirmar que a menor parte dos respondentes possui nível alto/moderado de conhecimento financeiro, pois apenas 17,24\% acertaram todas as questões. Os respondentes sem nenhum nível de conhecimento financeiro somaram 9,70\%. A maioria dos entrevistados, $73,06 \%$, foram considerados com baixo conhecimento financeiro.

O baixo nível de conhecimento financeiro encontrado nessa pesquisa está em consonância com outros estudos realizados e apresentados nesse trabalho. Nos últimos anos, diversos pesquisadores se dedicaram a investigar o nível de conhecimento financeiro, tanto de estudantes universitários quanto da população em geral, e vem obtendo resultados bastante negativos, como: Disney e Gathergood (2011) e Van Rooij, Lusardi e Alessie (2011).

Especialmente em relação a geração $\mathrm{Y}$, os resultados estão em coerência com as pesquisas analisadas que apontaram para a carência de conhecimento financeiro deste grupo (REED, 2008; LUSARDI; MITCHELL, 2010; CUDMORE et al., 2010). Os achados revelam uma necessidade de investimentos na educação financeira os jovens da geração Y, pois essa carência pode trazer impactos em diversos setores no decorrer da vida útil desses jovens e também em seu período de aposentadoria.

\subsection{Fatores determinantes do nível de conhecimento financeiro}

A verificação dos fatores determinantes do nível de conhecimento financeiro foi realizada através de análise multivariada pelo método de regressão logística binária. Foram considerados previamente os testes de validade do modelo estatístico, tendo sido encontrados resultados satisfatórios, como demonstra a tabela 4 . A tabela 5 apresenta os resultados obtidos pela regressão logística. 
Tabela 4 - Testes de Validade do Modelo Estatístico

\begin{tabular}{l|r}
\hline \multicolumn{1}{c|}{ Indicador } & \multicolumn{1}{c}{ Valor } \\
\hline Durbin-Watson & 1,856 \\
Hosmer and Lemeshow Test (sig) & 0,836 \\
Nagelkerke R Square & 0,097 \\
Predicted Overwall Percentage (Cut value=0,18) & 62,7 \\
VIF Idade & 1,004 \\
VIF Curso & 1,032 \\
VIF Gênero & 1,034 \\
\hline
\end{tabular}

Fonte: Elaborado pelos autores

Tabela 5 - Resultados Regressão Logística

\begin{tabular}{|c|c|c|c|c|c|c|c|c|}
\hline & & & & & & & $95 \%$ C.I.I & $\overline{\mathrm{EXP}(\mathrm{B})}$ \\
\hline & B & S.E. & Wald & df & Sig. & $\operatorname{Exp}(B)$ & Lower & Upper \\
\hline Idade & & & 2,171 & 3 & ,538 & & & \\
\hline Idade(1) & 19,156 & 28287,587 &, 000 & 1 & ,999 & 208632102,200 & 0,000 & \\
\hline Idade(2) & 19,184 & 28287,587 & ,000 & 1 & ,999 & 214457372,733 & 0,000 & \\
\hline Idade(3) & 19,646 & 28287,587 & ,000 & 1 & ,999 & 340665085,383 & 0,000 & \\
\hline Curso & & & 6,151 & 3 &, 105 & & & \\
\hline Curso(1) & ,618 & ,379 & 2,662 & 1 & , 103 & 1,855 & ,883 & 3,897 \\
\hline Curso(2) &, 113 & ,456 &, 061 & 1 & ,804 & 1,120 & ,458 & 2,738 \\
\hline Curso(3) &,- 118 & ,468 & ,064 & 1 & ,801 & ,889 & ,355 & 2,225 \\
\hline Gênero & 1,037 & ,263 & 15,538 & 1 &, 000 & 2,820 & 1,684 & 4,723 \\
\hline Constant & $-21,590$ & 28287,587 & ,000 & 1 & ,999 & ,000 & & \\
\hline
\end{tabular}

Fonte: Elaborado pelos autores

A tabela 5 revela que a variável gênero foi a única obteve significância, considerando o nível de 5\%. Tendo em vista a codificação 0 para sexo feminino e 1 para sexo masculino, nota-se que pertencer ao sexo masculino aumenta em 2,82 vezes a possiblidade do respondente possuir conhecimento financeiro alto/moderado. Esse resultado fortalece os dados apresentados na análise da distribuição das repostas e está de acordo com os estudos de Lusardi e Mitchell (2011b) e Van Rooij, Lusardi e Alessie (2011), entre outros. 


\section{CONSIDERAÇÕES FINAIS}

Ao abordar o nível de conhecimento financeiro encontrado entre os jovens da geração Y estudantes de um centro universitário na região sul de São Paulo, este trabalho evidenciou elementos semelhantes a trabalhos anteriores: a maioria dos respondentes acertou apenas uma ou duas questões do questionário $(76,1 \%)$, revelando nível de conhecimento financeiro baixo.

A análise das frequências relacionadas às respostas por grupos e os resultados obtidos pela regressão logística binária, considerando a influência das variáveis no nível de conhecimento financeiro, subsidiaram outras conclusões importantes. Como em outros estudos referenciados, comprovou-se um menor conhecimento financeiro das mulheres, o que foi evidenciado na distribuição das respostas e também na regressão logística. Esse resultado pode ser um fator de atenção para a sociedade, cabendo evidenciar as origens e natureza dessa diferença de conhecimento entre os gêneros. Uma possível causa pode ser a segregação de gêneros ocorrida por anos na sociedade, impedindo oportunidades iguais e impedindo as mulheres de estudar e acessar o mercado de trabalho. Esse legado pode estar sendo refletido na capacitação das mães em suas filhas pertencentes à geração Y. Estudos posteriores poderão revelar que mulheres das próximas gerações poderão obter melhor desempenho nesse quesito.

As variáveis curso e idade não apresentaram significância na regressão logística, mas na distribuição de resultados observou-se um melhor desempenho dos alunos do curso de administração. Esse resultado pode suscitar uma discussão. Se os cursos de Administração e Ciências Contábeis possuem um grande número de disciplinas em comum, cabe questionar as razões pelas quais os alunos de Administração obtiveram desempenho superior àquele. Isso é ainda mais intrigante quando se considera que o curso de Ciências Contábeis possui maior familiaridade com cálculos e controles financeiros e obteve um desempenho semelhante aos de Educação Física e Psicologia, que não possuem disciplinas na área financeira.

Vários itens poderiam ser analisados em maior profundidade para se descobrir as possíveis causas dessa discrepância e poderiam considerados em estudos posteriores, como as notas de entrada dos alunos nos respectivos cursos, as notas atuais, o ano de curso dos respondentes, a atividade profissional exercida.

Finalizando, destacam-se contribuições da pesquisa para as áreas acadêmica e profissional. Este estudo é útil por oferecer uma análise do nível de conhecimento financeiro dos jovens da geração Y no contexto da cidade de São Paulo. Podem se beneficiar dos resultados encontrados as instituições financeiras e de crédito, para moldar os produtos e serviços oferecidos a esse público; os governos, demonstrando a necessidade de orientar os

ReFAE - Revista da Faculdade de Administração e Economia, v. 8, n. 2, p. 17-36, 2017 
jovens nas questões financeiras; os jovens desta geração, alertando-os quanto a necessidade de ampliar suas bases de conhecimento financeiro; Empresas que oferecem produtos e serviços para os jovens.

Como limitação está o fato de ter-se trabalhado com apenas uma geração, em apenas uma região da cidade, estudar apenas um centro universitário e também a quantidade limitada de variáveis independentes consideradas. Sugere-se a aplicação mais ampla de questionários que considere outras gerações e possam ser comparados os resultados com estudos que tracem um perfil relacionado com renda, escolaridade, oportunidades de educação financeira em casa e tópicos relacionados. Podem ser empreendidos estudos em outros centros universitários e os resultados comparados com os obtidos nesta pesquisa para se analisar o nível de conhecimento financeiro em diferentes perfis de universitários.

\section{REFERÊNCIAS}

AGARWAL, S. et al. The age of reason: financial decisions over the life-cycle with implications for regulation. SSRN Working Paper Series, 2009.

ALLEN, P. Welcoming Y. Benefits Canada, Toronto, v. 28, n. 9, p. 51-53, Sept. 2004.

AVARD, S.; MANTON, E.; ENGLISH, D.; WALKER, J. The financial knowledge of college freshmen. College Student Journal. 39(02), 321-339, 2005.

BRASIL. Decreto $\mathrm{n}^{\mathrm{o}} 7.397$ de 22 de dezembro de 2010. Institui a Estratégia Nacional de Educação Financeira - ENEF, dispõe sobre a sua gestão e dá outras providências. Diário Oficial da União, Brasília, DF, 23 dez. 2010.

CENNAMO, C.; GARDNER, D. Generational differences in work values, outcomes and person-organisation values fit. Journal of Managerial Psychology. Bradford, v. 23, n. 8, p. 891-906, 2008.

CHEN, H.; VOLPE, R. P. Gender differences in personal financial literacy among college students. Financial Services Review, 11(03), 289-307, 2002.

CHINEN, K.; ENDO, H. Effects of attitude and background on personal financial ability: a student survey in the United States. International Journal of Management, Poole, v. 29, n. 1, p. 33-45, Mar. 2012.

CLAUDINO, L. P.; NUNES, M. B.; SILVA, F. C. Finanças pessoais: um estudo de caso com servidores públicos. ? In: SEMINÁRIOS EM ADMINISTRAÇÃO, 12., 2009, São Paulo. Anais. São Paulo: SEMEAD, 2012.

CORDEIRO, H. T. D. et al. A questão das gerações no campo da gestão de pessoas: tema emergente? In: SEMINÁRIOS EM ADMINISTRAÇÃO, 15, 2012, São Paulo. Anais. São Paulo: SEMEAD, 2012. 
CROSSAN, D.; FESLIER, D.; HURNARD, R. Financial literacy and retirement planning in New Zealand. Journal of Pension Economics \& Finance, Cambridge, v. 10, n. 4, p. 619635, Oct. 2011.

CUDMORE, B. A. et al. The millennials and money management. Journal of Management and Marketing Research, Jacksonville, v. 4, p. 1-28, Mar. 2010.

CULL, M.; WHITTON, D. University students' financial literacy levels: obstacles and Aids. The Economic and Labour Relations Review: ELRR, Sydney, v. 22, n. 1, p. 99-114, May 2011.

DINIZ, P. C. O. C.; PEREIRA, N. A.; ROGERS, P. A relação da educação financeira e do otimismo no número de cartões de crédito. In: SEMINÁRIOS EM ADMINISTRAÇÃO, 17. 2014, São Paulo. Anais. São Paulo: SEMEAD, 2014.

DISNEY, R.; GATHERGOOD, J. Financial literacy and indebtedness: new evidence for UK consumers. EconPapers, Discussion Papers, University of Nottingham, Centre for Finance, Credit and Macroeconomics (CFCM). 2011.

FÁVERO, L. P.; BELFIORE, P.; SILVA, F. L.; CHAN, B. L. Análise de Dados: modelagem multivariada para tomada de decisões. Rio de Janeiro: Elsevier, 2009.

FILENGA, D.; VIEIRA, A. M. Notas sobre o trabalho e seu contexto social. Revista UNIABEU, v. 5, p. 1-16, 2012.

GIL, A. C. Como elaborar projetos de pesquisa. 4. ed. São Paulo: Atlas, 2002.

HAIR JR., J. F. et al. Análise multivariada de dados. 5. ed. Porto Alegre: Bookman, 2005.

HEANEY, J. Generations $\mathrm{X}$ and Y's internet banking usage in Australia. Journal of Financial Services Marketing, v. 11, n. 3, p. 196-210, Feb. 2007.

HERBIG, P. A.; BORSTORFF, P. Japan's Shinjinrui: the new breed. International Journal of Social Economics, Bradford, v. 22, n. 12, p. 49-65, 1995.

HILGERT, M. A.; HOGARTH, J. M.; BEVERLY, S. G. Household financial management: the connection between knowledge and behavior. Federal Reserve Bulletin, Washington, v. 89, n. 7, p. 309, July, 2003.

HUSTON, S. J. Measuring financial literacy. The Journal of Consumer Affairs, Madison, v. 4, n. 2, p. 296-316, 2010.

KLAPPER, L.; LUSARDI, A.; PANOS, G. A. Financial literacy and the financial crisis: evidence from Russia. SSRN Working Paper Series., 2011.

LUCCI, C. R.; ZERRENER, S. A.; VERRONE, M. A. G.; SANTOS, S. C. A Influência da Educação Financeira nas Decisões de Consumo e Investimento dos Indivíduos. In: SEMINÁRIOS EM ADMINISTRAÇÃO, 09, 2006, São Paulo. Anais. São Paulo: SEMEAD, 2006. 
LUSARDI, A. Financial Literacy: an essential tool for informed consumer choice? Paolo Baffi Centre Research Paper Series, n. 2009-35, 2008.

; MITCHELL, O. S. Financial literacy and planning: implications for retirement wellbeing. Oct. Pension Research Council Working Paper, n. 1, 2006.

Baby boomer retirement security: the roles of planning, financial literacy, and housing wealth. Journal of Monetary Economics, Amsterdam, v. 54, n. 1, p. 205-224, Jan. 2007.

Planning and financial literacy: how do women fare? The American Economic Review. Nashville, v. 98, n. 2, p. 413-417. May 2008.

; Financial literacy and retirement planning in the United States. Journal of Pension Economics \& Finance, Cambridge, v. 10, n. 4, p. 509-525, Oct. 2011a.

; Financial literacy around the world: an overview. Journal of Pension Economics \& Finance, Cambridge, v. 10, n. 4, p. 497-508, Oct. 2011 b.

; _ The economic importance of financial literacy: theory and evidence. Journal of Economic Literature, Nashville, v. 52, n. 1, p. 5-44, Mar. 2013.

implications for consumer policy. Nov. SSRN Working Paper Series, 2010.

; TUFANO, P. Teach workers about the perils of debt. Harvard Business Review, Boston, v. 87, n. 11, p. 22-24, Nov. 2009.

LYONS, A. C. A profile of financially at-risk college students. The Journal of Consumer Affairs, 38, 56-80. 2004.

LYONS, A. C.; CHANG, Y. H.; SHERPF, E. Translating financial education into behavior change for low-income populations. Financial Counseling \& Planning, 17(2). 27-45. 2006.

LYONS, A. C.; PALMER, L.; JAYARATNE K.; SHERPF, E. Are We Making the Grade? A National Overview of Financial Education and Program Evaluation. The Journal of Consumer Affairs, 40 (2): 208-235. 2006.

MANDELL, L. Financial Literacy in High School. In Overcoming the Saving Slump: How to Increase the Effectiveness of Financial Education and Saving Programs, edited by Annamaria Lusardi (257-279). Chicago: University of Chicago Press, 2008.

MANDELL, L.; KLEIN, L. S. The impact of financial literacy education on subsequent financial behavior. Journal of Financial Counseling and Planning, Columbus, v. 20, n. 1, p. 15-24, 2009.

MURPHY, D. S.; YETMAR, S. Personal financial planning attitudes: a preliminary study of graduate students. Management Research Review, 33(08), 811-817, 2010 
NAYYAR, S. Inside the mind of Gen Y. American Demographics, Detroit, v. 23, n. 9, p. 6, Sept., 2001.

NEIDERMEYER, A. A.; NEIDERMEYER, P. E. The missing curriculum link: personal financial planning. American Journal of Business Education, 3(04), 79-82, 2010.

NGA; J. K. H.; YONG; L. H. L.; SELLAPPAN, R. D. A study of financial awareness among youths. Young Consumers, Bradford, v. 11, n. 4, p. 277-290, 2010.

NORVILITIS, J. M. et al. Personality factors, money attitudes, financial knowledge, and credit-card debt in college students. Journal of Social Psychology, 36(6), 1395-1413, 2006.

NORVILITIS, J. M.; SZABLICKI, P. B.; WILSON, S. D. Factors influencing levels of credit card debt in college students. Journal of Applied Social Psychology, 33(5), 935-947, 2003.

ORGANIZAÇÃO PARA COOPERAÇÃO E DESENVOLVIMENTO ECONÔMICO (OCDE). Recommendation on principles and good practices for financial education and awareness. July 2005. Disponível em: < http://www.oecd.org/finance/financialeducation/35108560.pdf>. Acesso em: jun. 2016.

ORGANIZAÇÃO PARA COOPERAÇÃO E DESENVOLVIMENTO ECONÔMICO (OCDE). Measuring financial literacy: core questionnaire in measuring financial literacy: questionnaire and guidance notes for conducting an internationally comparable survey of financial literacy. Paris, 2011.

PORTRICH, A. C. G.; VIEIRA, K. M.; CERETTA, P. S. Nível de alfabetização financeira dos estudantes universitários: afinal, o que é relevante? Revista Eletrônica de Ciência Administrativa, Campo Largo, v. 12, n. 3, p. 314-333, set. 2013.

REED, M. Student debt and the class of 2007. [S. 1.]: The Project on Student Debt, 2008. Disponível em: <http://projectonstudentdebt.org/files/pub/classof2007.pdf>. Acesso em: 15 jun. 2016.

REMUND, D. L. Financial literacy explicated: the case for a clearer definition in an increasingly complex economy. The Journal of Consumer Affairs, Madison, v. 44, n. 2, p. 276-295, 2010.

ROBERTS, J .A.; JONES, E. Money attitudes, credit card use, and compulsive buying among college students, The Journal of Consumer Affairs, Madison, v. 35, n. 2, p. 213-240, 2001.

ROGERS, P.; FAVATO, V.; SECURATO, J. R. Efeito educação financeira no processo de tomada de decisões em investimentos: um estudo a luz das finanças comportamentais. In: II Congresso ANPCONT-Associação Nacional dos Programas de Pós-Graduação em Ciências Contábeis, Salvador/BA. 2008.

SAVOIA, J. R. F.; SAITO, A. T.; SANTANA, F. A. Paradigmas da educação financeira no Brasil. Revista de Administração Pública, Rio de Janeiro, v. 41, n. 6, p. 1121-1141, nov./dez. 2007. 
SCHFFMAN, L. G.; KANUK, L. L. Comportamento do consumidor. 9. ed. Rio de Janeiro: LTC, 2009.

SHRYK. Shryk needs to understand the market IT is targeting. 2008. Alarm: Clock.com [Weblog post]. Disponível em: http://www.thealarmclock.com. Acesso em 15 de jun. 2014.

TANG, T. L. The meaning of money revisited. Journal of Organizational Behavior, Chichester, v. 13, n. 2, p. 197-202, 1992.

TWENGE, J. M.; CAMPBELL, S. M. Generational differences in psychological traits and their impact on the workplace. Journal of Managerial Psychology, Bradford, v. 23, n. 8, p. 862-877, 2008.

VAN ROOIJ, M.; LUSARDI, A.; ALESSIE, R. J. M. Financial literacy and stock market participation. Oct., SSRN Working Paper Series, 2011.

VIEIRA, S. F. A.; BATAGLIA, R. T. M.; SEREIA, V. J.; RIBEIRO, M. L.; LOHMANN, G. Educação financeira e decisões de consumo, investimento e poupança: uma análise dos alunos de uma universidade pública do Paraná? In: SEMINÁRIOS EM ADMINISTRAÇÃO, 15., 2012, São Paulo. Anais. São Paulo: SEMEAD, 2012.

WILLIAMS, K. C.; PAGE, R. A. Marketing to the generations. Journal of Behavioral Studies in Business, Jacksonville, v. 3, p. 1-17, Apr., 2011. 